www.mdpi.com/journal/challenges

Article

\title{
Assessing the Possibility of Biological Complexity on Other Worlds, with an Estimate of the Occurrence of Complex Life in the Milky Way Galaxy
}

\section{Louis N. Irwin ${ }^{1, *}$, Abel Méndez ${ }^{2}$, Alberto G. Fairén ${ }^{3,4}$ and Dirk Schulze-Makuch ${ }^{5,6}$}

1 Department of Biological Sciences, University of Texas at El Paso, 500 W. University Avenue, El Paso, TX 79968, USA

2 Planetary Habitability Laboratory, University of Puerto Rico at Arecibo, Arecibo, Puerto Rico 00163, USA; E-Mail: abel.mendez@upr.edu

3 Department of Astronomy, Cornell University, 426 Space Science Bldg., Ithaca, NY 14853, USA;

E-Mail: agfairen@cornell.edu

4 Centro de Astrobiología, CSIC-INTA, M-108 Km 4, Torrejón de Ardoz 28850, Spain

5 School of the Environment, Washington State University, Pullman, WA 99164, USA; E-Mail:dirksm@wsu.edu

6 Center of Astronomy and Astrophysics, Technical University Berlin, Berlin 10623, Germany

* Author to whom correspondence should be addressed; E-Mail: lirwin@utep.edu; Tel: +1-915-497-7267; Fax: +1-303-623-4317.

Received: 19 March 2014; in revised form: 19 May 2014 / Accepted: 20 May 2014 / Published: 28 May 2014

Abstract: Rational speculation about biological evolution on other worlds is one of the outstanding challenges in astrobiology. With the growing confirmation that multiplanetary systems abound in the universe, the prospect that life occurs redundantly throughout the cosmos is gaining widespread support. Given the enormous number of possible abodes for life likely to be discovered on an ongoing basis, the prospect that life could have evolved into complex, macro-organismic communities in at least some cases merits consideration. Toward that end, we here propose a Biological Complexity Index (BCI), designed to provide a quantitative estimate of the relative probability that complex, macro-organismic life forms could have emerged on other worlds. The BCI ranks planets and moons by basic, first-order characteristics detectable with available technology. By our calculation only 11 $(\sim 1.7 \%)$ of the extrasolar planets known to date have a BCI above that of Europa; 
but by extrapolation, the total of such planets could exceed 100 million in our galaxy alone. This is the first quantitative assessment of the plausibility of complex life throughout the universe based on empirical data. It supports the view that the evolution of complex life on other worlds is rare in frequency but large in absolute number.

Keywords: exoplanets; biocomplexity; evolution; habitability

\section{Introduction}

While life is known to exist with certainty only on Earth, there are compelling reasons for assuming that it could exist throughout the universe in abundance [1-3]. Organic molecules have been found in star forming regions [4], around protoplanetary disks [5], in meteorites [6], in comets [7], and in deep space [8]. Water is among the most common molecules in the universe, and a host of other liquids can exist at planetary temperatures [9]. Besides an abundance of light and heat in all stellar systems, many other forms of energy are locally available on probably most planetary bodies [10-14]. Thus, the prerequisites for life are commonplace throughout the cosmos.

In response to the intuitive logic that life is likely to be found on other worlds like the one world where it is known to exist, the search for other worlds that could harbor life has emphasized searching for planets similar to Earth in geophysical properties and in relation to their central stars. A growing list of such planets has been confirmed [15], including Gl581c and d [16,17], GJ667Cc [18-20], Kepler-62e and f [21], HD40307g [22], and HD85512b [23]. Atmospheric modeling studies have been conducted for both G1581d and HD85512b [24-27], confirming the potential habitability of the former. As the number of known exoplanets has grown, the need for quantitative measures of their similarity to Earth has become apparent. As one approach to meeting this need, we have proposed an Earth Similarity Index, or ESI, which rates the similarity of extrasolar planets to Earth on the basis of mass, size, and temperature [28].

There is also, however, broad if not universal agreement that life could exist in forms quite dissimilar from those on Earth [3,29,30]. Even on our planet, organisms live in a range of environmental conditions so broad [31,32] that the possibility of life under the most extreme conditions by terran standards cannot be ruled out. Other planets could harbor forms of life unknown to us, or forms quite extremophilic in relation to life on Earth, under conditions and on planetary bodies very different from Earth. To address this possibility, we have also proposed a metric for assessing habitability in a generic and non-terracentric sense. Our Planetary Habitability Index, or PHI, thus, provides a second tier of analysis for the search for life on other worlds [28].

A question that inevitably arises is whether, and to what extent, life on other worlds can and has evolved to a level of size, diversity, and complexity known on Earth, or even beyond those levels. While statistical arguments have been advanced that the relatively early appearance of life in the history of the Earth supports the likelihood that life could arise elsewhere under similar circumstances [33], statistical confidence in predicting the future course of life is much lower [34]. The Rare Earth Hypothesis [35] states that even on planets that resemble the Earth geophysically, a host of other conditions have coincided to make complex life more likely to arise on Earth than on the vast majority of exoplanets. 
Though the requirements for habitability as reflected in the PHI are necessary, they are not sufficient for identifying those worlds most likely to be amenable to the evolution of a higher degree of biological complexity. Another metric is required for this third level of assessing the probability of finding complex life in the universe. For this purpose, we here propose a Biological Complexity Index (BCI), as a complement to the ESI and PHI, in order to provide a more complete and nuanced predictor of where and in what forms life might be expected to exist on other worlds. The BCI differs from the PHI, in that it estimates a subset of those worlds on which any form of life might appear. The BCI differs from the ESI by ranking planets on their habitability for complex biology, rather than their geophysical similarity to Earth. All three metrics employ some overlapping planetary attributes, but the predictive value of each is geared toward a different objective. To our knowledge, this is the first systematic attempt to assess the relative possibility of the evolution of complex forms of life on Solar System bodies and exoplanets on the basis of empirically observable and quantifiable metrics rather than qualitative or purely hypothetical arguments.

\subsection{Rationale}

\subsubsection{Definition}

The simplest microbe is a highly complex structural, chemical, and informational system. As used in this paper, however, complexity refers to the overall biota of a planetary body that is diverse in size (including macroorganismic), form, history, and distribution. This definition doesn't require that organisms be large or active, like animals on Earth, but it does intend to include ecosystems consisting of a variety of organisms in multiple trophic levels that occupy a variety of ecological niches and a range of life histories - in other words, a biosphere that has undergone evolutionary diversification beyond the level of mere microbial differentiation.

\subsubsection{Parameters Relevant to Biocomplexity}

Those planetary characteristics most likely to promote the evolution of complex forms of life have been described and defended in detail previously [2,3]. In brief summary, the most salient factors are the availability of abundant energy, extensive habitat fractionation through topographical complexity and multiple habitat layers, freedom from sequestration, latitudinal zonation and seasonal cycles, long-term environmental changes of the type generated on a geologically active world and embodied by a complex and lengthy planetary history.

Combining the requirements for life in general with the properties most likely to favor the evolution of complex life boils down to seven fundamental characteristics. Four of these-substrate, energy, chemistry, and liquid-are broadly recognized, as previously described [28]. The three added planetary characteristics that favor the evolution of complex life are the following:

Geophysics (G). A planet's geophysical differentiation provides the opportunity for multiple habitats and interfaces between those habitats. The higher the density of a planetary body, the more likely it is to be rocky and differentiated into a core, mantle, and crust [36-38]. Above a certain mass, it is more likely to still be heated internally by radioactive decay, and to have plate tectonics, especially if the water content of the planet is substantial [39], 
which would lead to topographical complexity and geochemical cycling [40]. It also is more likely to interact gravitationally with other massive bodies nearby, and to have a more complex planetary history. Density alone does not guarantee any of these things, and different models lead to different predictions $[38,41]$. But without a significant density, the planet, if large, is likely to be a gas giant with no solid substrates or differentiation, and if small, is probably an undifferentiated snowball satellite. In both cases, their planetary histories are likely to be simple.

Orbital properties determine seasonal cycles, which, together with latitudinal variations, generate ecological diversity and spatiotemporal changes in climate. Eccentric orbits provide different degrees of stellar flux at apoastron and periastron. This, along with obliquity, contributes to seasonal cycles that spur the adaptive modifications of natural selection to different degrees in different organisms, generating diversity. While data on obliquity are available only for Solar System objects, orbital eccentricity is established for about $90 \%$ of the known exoplanetary inventory.

Temperature (T). As in all aspects of biology, temperature is a critical variable. Temperatures have to be warm enough for biochemical reactions to occur at a feasible pace, but not so hot that macromolecular structure cannot be maintained. They have to be warm enough to enable freedom of motion and growth in size in a liquid medium or on a solid surface, but cool enough to allow for hydrological (or analogous) cycling.

The presence of liquid is assumed to be a requirement on any planetary body on which living organisms have evolved. Therefore, temperatures obviously have to fall within the range at which a solvent can exist in liquid form. Furthermore, complex forms of life on Earth require a narrower range of temperatures than those at which unicellular life can exist-probably because of factors related to heat distribution in larger bodies, and other physiological and mechanical constraints. Therefore, complex life is most likely to evolve on planets that maintain temperatures within a particular optimal range, though no empirical evidence is available on what that optimum is, apart from the case of life as we know it on Earth.

Age (A). Evolution operates through a temporal dimension. While the extent to which time is required for any given stage of evolution to be reached is far from certain, the empirical observation from life on Earth is that major evolutionary steps, such as the appearance of multicellularity and the emergence of oxidative respiration, occurred over billion-year time scales. For very old planets, exhaustion of internally generated heat may result in eventual cooling, with consequences for global temperatures and atmospheric composition. However, most exoplanets discovered to date are larger than Earth, and Earth is still generating internal heat, so our simplifying assumption is that evolutionary trajectories for the exoplanets known to date can logically be expected to be more elaborate on bodies that have had a longer planetary history.

\subsection{Components of the Biological Complexity Index (BCI)}

Combining the four essential properties for the support of life in general-substrate, energy, chemistry, and liquid-with the three added properties of geophysical complexity, appropriate temperature, and age, leads to seven parameters relevant to the evolution of biological complexity. Unfortunately, a chemical profile of bodies at exoplanetary distances is not yet available with current technology; so we do not consider chemistry in the calculation of the BCI at this time. 
As the chemical composition of exoplanets becomes known, it should be factored in to any calculation purporting to predict biological complexity. Neither is information about liquids directly available for any exoplanet, though thermal data, either modeled or directly observed, can serve as a proxy for the possible presence of liquids. Thus, the five factors remaining for computation of a BCI are substrate $(S)$, energy (E), geophysics (G), temperature (T), and age (A). Our approach is to develop quantitative metrics for each of these parameters, based on directly observable of objectively modeled data, and to calculate the geometric mean of the five parameters taken together to yield the BCI. By dividing the absolute $\mathrm{BCI}\left(\mathrm{BCI}_{a b s}\right)$ for any planetary body by the maximum $\mathrm{BCI}_{a b s}$ in the data set, a relative $\mathrm{BCI}$ $\left(\mathrm{BCI}_{r e l}\right)$, which scales from 0 to 1 , is obtained.

\section{Results and Discussion}

Of the 637 exoplanets for which data on all relevant parameters are available, 364 (57\%) yield $\mathrm{BCI}$ values $>0$. The mean $\mathrm{BCI}_{\text {rel }}$ value is 0.50 (median $=0.52$ ), which falls slightly below that for Saturn.

Exoplanets for which the $\mathrm{BCI}_{r e l}>0.50$ are listed in Table 1, along with equivalently ranked Solar System bodies. The highest BCI value is for Gliese 581c, followed by Earth, followed by HD 85512b, HD 20794d, Kepler-20d, and Gliese 581d, then by Mars, five more exoplanets, and Europa. Of the 637 exoplanets in the sample here considered, $10(1.6 \%)$ have BCI values higher than for Europa, and five $(0.8 \%)$ have BCI values higher than for Mars.

Six of the exoplanets with the 14 highest BCI values orbit their central stars within the habitable zone as conventionally defined. However, four of the top five BCI values belong to exoplanets that orbit outside their stellar habitable zones.

Gliese 581c, orbits slightly inside the inner limits of a habitable zone around a star one-third as massive as the Sun. Its mass is six times greater than Earth's but with a density $31 \%$ lower. Its equilibrium temperature is estimated to be $39^{\circ} \mathrm{C}$, with an atmospheric pressure of $4.3 \mathrm{bar}$.

HD $85512 \mathrm{~b}$ is four times more massive than Earth, orbiting a star $31 \%$ less massive than the Sun. It has an equilibrium temperature estimated to be $24{ }^{\circ} \mathrm{C}$ with an atmospheric pressure of 3.3 bar.

HD20794d, the outermost of three known planets orbiting a star somewhat smaller than the Sun has an estimated equilibrium temperature of $115{ }^{\circ} \mathrm{C}$ and an atmospheric pressure of 4 bar. Compared to Earth, its mass is 5.5 times greater but with a density $27 \%$ less.

Kepler-20d orbits a star only $9 \%$ less massive than the Sun. Its mass is almost 10 times that of Earth's but its density is less than half. It has a highly eccentric orbit, so its estimated equilibrium temperature oscillates between $42{ }^{\circ} \mathrm{C}$ and $357^{\circ} \mathrm{C}$, with a mean of $115^{\circ} \mathrm{C}$, under a pressure of $4.1 \mathrm{bar}$.

Kepler-20d is most likely a water world, while the other three are presumed to be rocky-water planets. All five exoplanets with higher BCI values than for Mars orbit stars older than the Sun (more than three times older, for HD20794d). It is this factor, plus their abundance of energy, that gives these bodies such high BCI values.

These characteristics describe super-terran planets that could be largely water worlds with a long time for evolutionary scenarios to play out. They may have been cooler at younger ages. As they warmed, complex ecosystems could either have perished or evolved by gradual adaptation to higher temperatures in water kept liquid by high atmospheric pressure and/or high pressure at ocean depths. 
The recent discovery of Kepler-186f [42] has drawn attention as an Earth-sized exoplanet orbiting within the stellar habitable zone of Kepler-186. It ranks 12th in its BCI value, slightly above that of Europa (Table 1, Figure 1). This BCI value is relatively low, despite its similarity in size to Earth, because it receives substantially less solar energy, is cooler, and appears to be somewhat younger than Earth.

Table 1. Known planetary bodies, as of 14 January 2014 (with addition of Kepler-186f), ranked in descending order of their relative Biological Complexity Index $\left(\mathrm{BCI}_{r e l}\right)$, based on factors affecting the development of complex life. Solar System bodies and their ranking are included for comparison.

\begin{tabular}{|c|c|c|c|c|c|c|c|c|c|c|c|c|}
\hline \multirow[b]{2}{*}{ Rank } & \multirow[b]{2}{*}{ Body } & \multicolumn{2}{|c|}{ Substrate $^{\mathrm{a}}$} & \multicolumn{2}{|c|}{ Energy $^{b}$} & \multicolumn{2}{|c|}{ Temperature $^{c}$} & \multicolumn{2}{|c|}{ Geophysics $^{\mathrm{d}}$} & \multirow[b]{2}{*}{$\operatorname{Age}^{e}$} & \multirow{2}{*}{$\begin{array}{l}\text { BCI } \\
a b s^{\mathrm{f}}\end{array}$} & \multirow{2}{*}{$\begin{array}{l}\mathrm{BC} \\
\mathrm{rel}\end{array}$} \\
\hline & & Layers & $\begin{array}{c}\text { Atmospheric } \\
\text { Complexity }\end{array}$ & $\begin{array}{c}\text { Stellar } \\
\text { Flux } \\
\end{array}$ & $\begin{array}{l}\text { Redox } \\
\text { Molecs } \\
\end{array}$ & $\begin{array}{l}\text { Sub- } \\
\text { surf }\end{array}$ & Surf & Density & $\begin{array}{l}\text { Eccen- } \\
\text { tricity }\end{array}$ & & & \\
\hline 1 & Gl 581c & 3 & 2 & 0.86 & 1 & 1.00 & 0.87 & 0.83 & 0.07 & 1.78 & 1.95 & 1.00 \\
\hline 2 & Earth & 3 & 2 & 0.50 & 2 & 0.92 & 0.92 & 1.00 & 0.02 & 1.00 & 1.88 & 0.97 \\
\hline 3 & HD $85512 b$ & 3 & 2 & 0.94 & 1 & 0.95 & 0.92 & 0.91 & 0.11 & 1.25 & 1.87 & 0.96 \\
\hline 4 & HD $20794 d$ & 3 & 2 & 0.00 & 1 & 0.76 & 0.63 & 0.85 & 0.00 & 3.11 & 1.79 & 0.92 \\
\hline 5 & Kepler-20d & 3 & 2 & 0.00 & 1 & 0.75 & 0.63 & 0.66 & 0.60 & 1.96 & 1.76 & 0.91 \\
\hline 6 & Gl 581d * & 3 & 2 & 0.13 & 1 & 0.58 & 0.71 & 0.81 & 0.20 & 1.78 & 1.67 & 0.86 \\
\hline 7 & Mars & 3 & 2 & 0.22 & 1.5 & 0.67 & 0.67 & 0.84 & 0.09 & 1.00 & 1.61 & 0.83 \\
\hline 8 & GJ $163 c *$ & 3 & 2 & 0.70 & 1 & 0.89 & 0.98 & 0.77 & 0.09 & 0.67 & 1.56 & $\mathbf{0 . 8 0}$ \\
\hline 9 & HD $20794 c$ & 2 & 2 & 0.00 & 1 & 0.37 & 0.25 & 0.95 & 0.00 & 3.11 & 1.49 & 0.76 \\
\hline 10 & Gl 581e & 2 & 2 & 0.00 & 1 & 0.39 & 0.27 & 0.97 & 0.32 & 1.78 & 1.44 & 0.74 \\
\hline 11 & Kepler-10c & 3 & 2 & 0.00 & 1 & 0.34 & 0.21 & 0.79 & 0.00 & 2.64 & 1.41 & 0.73 \\
\hline 12 & Kepler-186f* & 2 & 2 & 0.16 & 1 & 0.62 & 0.73 & 0.95 & 0.00 & 0.89 & 1.39 & 0.72 \\
\hline 13 & Kepler-37d & 3 & 2 & 0.00 & 1 & 0.53 & 0.40 & 0.85 & 0.00 & 1.33 & 1.39 & 0.72 \\
\hline 14 & Europa & 3 & 2 & 0.02 & 1.5 & 0.51 & 0.33 & 0.76 & 0.05 & 1.00 & 1.39 & 0.71 \\
\hline 15 & GJ $667 \mathrm{Cc}$ * & 3 & 2 & 0.44 & 1 & 0.79 & 0.92 & 0.89 & 0.02 & 0.44 & 1.38 & 0.71 \\
\hline 16 & GJ $667 \mathrm{Cf} *$ & 3 & 2 & 0.28 & 1 & 0.71 & 0.83 & 0.94 & 0.03 & 0.44 & 1.33 & 0.69 \\
\hline 17 & Titan & 3 & 2 & 0.01 & 1.5 & 0.43 & 0.43 & 0.57 & 0.06 & 1.00 & 1.32 & 0.68 \\
\hline 18 & Io & 2 & 2 & 0.02 & 1 & 0.52 & 0.52 & 0.80 & 0.05 & 1.00 & 1.29 & 0.67 \\
\hline 19 & Kepler-61b * & 3 & 2 & 0.64 & 1 & 0.86 & 0.99 & 0.82 & 0.25 & 0.22 & 1.29 & 0.66 \\
\hline 20 & GJ $667 \mathrm{Ce}$ * & 3 & 2 & 0.15 & 1 & 0.61 & 0.73 & 0.94 & 0.02 & 0.44 & 1.27 & 0.65 \\
\hline 21 & GJ 1214b & 3 & 2 & 0.00 & 1 & 0.33 & 0.21 & 0.58 & 0.27 & 1.33 & 1.26 & 0.65 \\
\hline 22 & GJ $667 \mathrm{Cb}$ & 3 & 2 & 0.00 & 1 & 0.76 & 0.63 & 0.82 & 0.13 & 0.44 & 1.24 & 0.64 \\
\hline 23 & Callisto & 3 & 2 & 0.02 & 1 & 0.43 & 0.43 & 0.57 & 0.05 & 1.00 & 1.22 & 0.63 \\
\hline 24 & GJ $667 \mathrm{Cd}$ & 3 & 2 & 0.09 & 1 & 0.53 & 0.66 & 0.84 & 0.03 & 0.44 & 1.20 & 0.62 \\
\hline 25 & Ganymede & 3 & 2 & 0.02 & 1 & 0.35 & 0.35 & 0.59 & 0.05 & 1.00 & 1.18 & 0.61 \\
\hline 27 & Triton & 3 & 2 & 0.00 & 1 & 0.12 & 0.12 & 0.62 & 0.01 & 1.00 & 0.94 & 0.48 \\
\hline 28 & GJ $667 \mathrm{Cg}$ & 3 & 1 & 0.02 & 0.5 & 0.38 & 0.39 & 0.86 & 0.08 & 0.44 & 0.92 & 0.48 \\
\hline 29 & Moon & 1 & 0 & 0.50 & 0 & 0.39 & 0.55 & 0.78 & 0.02 & 1.00 & 0.82 & 0.42 \\
\hline
\end{tabular}


Table 1. Cont.

\begin{tabular}{|c|c|c|c|c|c|c|c|c|c|c|c|c|}
\hline \multirow[b]{2}{*}{ Rank } & \multirow[b]{2}{*}{ Body } & \multicolumn{2}{|c|}{ Substrate $^{a}$} & \multicolumn{2}{|c|}{ Energy $^{b}$} & \multicolumn{2}{|c|}{ Temperature $^{c}$} & \multicolumn{2}{|c|}{ Geophysics $^{\text {d }}$} & \multirow[b]{2}{*}{ Age $^{e}$} & \multirow{2}{*}{$\begin{array}{l}\text { BCI } \\
a b s^{\mathrm{f}}\end{array}$} & \multirow[b]{2}{*}{$\begin{array}{l}\mathrm{BCI} \\
\text { rel }^{\mathrm{g}}\end{array}$} \\
\hline & & Layers & $\begin{array}{c}\text { Atmospheric } \\
\text { Complexity }\end{array}$ & $\begin{array}{c}\text { Stellar } \\
\text { Flux }\end{array}$ & $\begin{array}{l}\text { Redox } \\
\text { Molecs }\end{array}$ & $\begin{array}{l}\text { Sub- } \\
\text { surf }\end{array}$ & Surf & Density & $\begin{array}{l}\text { Eccen- } \\
\text { tricity }\end{array}$ & & & \\
\hline 30 & $\begin{array}{c}\text { PSR } \\
125712 \mathrm{~d} \\
\end{array}$ & 3 & 2 & 0.00 & 1 & 0.18 & 0.06 & 0.92 & 0.03 & 0.22 & 0.76 & 0.39 \\
\hline 31 & Gl 876d & 3 & 2 & 0.00 & 1 & 0.04 & 0.00 & 0.82 & 0.21 & 0.56 & 0.73 & 0.33 \\
\hline 33 & Enceladus & 2 & 0 & 0.01 & 0 & 0.30 & 0.30 & 0.44 & 0.06 & 1.00 & 0.32 & 0.17 \\
\hline
\end{tabular}

Notes: * Planets orbiting their central stars within a conventionally defined habitable zone; ${ }^{\text {a }}$ Substrate (none assumed for planetary compositions of gas only): Layers: Solid ("rocky" or "rocky-iron") or gaseous only =1; solid with atmosphere or liquid $=2$; solid with atmosphere and liquid, or liquid between two solid layers $=3$; Atmospheric Complexity: $\mathrm{H}$ only $=1$; "metals-rich" $=2 ;{ }^{\mathbf{b}}$ Energy: Stellar Flux $(\mathrm{F})$ : Intensity of radiation from central star, expressed as a range from 0 to 1, with the optimum assumed to be 2 times the stellar flux for Earth, with an upper tolerance limit of 4 times Earth's stellar flux. The factor for energy derived from solar flux $=1-\left|1-\left(\mathrm{F}_{E} / 2\right)\right|$ Redox Molecules: "hydrogen-rich" atmosphere $=0.5$, "metals-rich" atmosphere $=1$, presence of oxidizer and/or reducer both suspected $=1.5$, presence of both confirmed $=2 ;^{\mathbf{c}}$ Temperatures: Both surface and subsurface temperatures $(\mathrm{T})$ are expressed by a factor ranging from 0 to 1 , where a value of 1 is equivalent to the most optimal temperature, here assumed to be $312{ }^{\circ} \mathrm{K}$, with an upper limit of $624^{\circ} \mathrm{K}$ for the survivability of complex life. The factor for thermal optimality is thus calculated to be $=1-\mid 1-(\mathrm{T} / 312)$; ${ }^{\mathrm{d}}$ Geophysics: Density: square root of density, normalized to Earth's density; Eccentricity: absolute value; ${ }^{\mathbf{e}}$ Age: Estimated age of central star, normalized to age of Solar System; ${ }^{\mathbf{f}}$ The $\mathrm{BCI}_{a b s}$ is the geometric mean of the value or sum of values for Substrate, Energy, Temperature, Geophysics, and Age (Equation 1 in section 3.2); ${ }^{\mathbf{g}} \mathrm{The} \mathrm{BCI}_{\text {rel }}$ is the ratio of the $\mathrm{BCI}_{a b s}$ to the largest $\mathrm{BCI}_{a b s}$ value in the data set.

Heller and Armstrong [43] have recently pointed to the likelihood that some exoplanets may be more optimally suited for life than Earth; hence the fact that at least one exoplanet rates higher than Earth (as it would be detected from a distance) is not surprising. According to these authors, such "superhabitable" worlds would likely be larger, warmer, and older, orbiting dwarf stars. All five exoplanets with BCI values higher than that for Mars have exactly those characteristics. Nine of the 10 exoplanets with the highest BCI ratings orbit stars older and smaller than the Sun and have higher estimated planetary temperatures than Earth. It is important to bear in mind that conditions deemed optimal for life on Earth are those under which life has evolved on this planet. Prolonged evolution under substantially different conditions could lead through natural selection to substantially different forms of life. Thus, while the current temperature on HD 20794d (115 ${ }^{\circ} \mathrm{C}$ equilibrium, $155{ }^{\circ} \mathrm{C}$ surface) is extreme by terran standards, a period of prolonged evolution - three times longer than life has had to evolve on Earth - could have either adapted complex organisms to those extremes, or at least passed through a cooler period during which complex life could have thrived.

Figure 1 plots the $\mathrm{BCI}_{r e l}$ as a function of the ESI for all planetary and solar system bodies with a $\mathrm{BCI}>0$, including gas giant planets, to allow comparison between the metrics for similarity to Earth and the possibility of the evolution of complex life. The plot reveals a highly significant correlation $\left(r^{2}=0.43, p<0.0001\right)$ among exoplanets between the two metrics.

The correlation between the BCI and ESI shown in Figure 1 is not surprising. Since we know that complex life has evolved on Earth, we can assume that it could evolve on planetary bodies similar to Earth, which is what the ESI measures. However, 13 non-gaseous exoplanets and all Solar System 
bodies except for Venus, Earth, and Mars have higher BCI values but lower ESIs than Mercury; i.e., are less similar to Earth than Mercury but have a greater potential for biological complexity. It could be argued that this results from criteria for the BCI that are less constraining than the assumptions underlying other concepts used to define habitability, such as the stellar habitable zone [44]. Indeed, only 10 exoplanets (as of 14 January 2014), were listed as residing within the habitable zone of their central stars as conventionally defined [15]. The possibility of life outside habitable zones remains controversial [3,45]. Until that issue is settled, a less rigid set of criteria, as embodied in the calculation for the BCI proposed here, seems warranted. It follows that the BCI embraces a greater range of possible forms that life can take than just the forms in which it exists on Earth.

Figure 1. Biological complexity $\left(\mathrm{BCI}_{r e l}\right)$ relative to Earth similarity (ESI), as calculated in Schulze-Makuch et al. [28], for Solar System planets (orange squares) and satellites (yellow squares), and for 365 exoplanets for which $\mathrm{BCI}_{r e l}>0$. The vast majority of exoplanets known to date are gas giants (green circles), but the ones with highest BCI values are rocky-water worlds (purple circles).

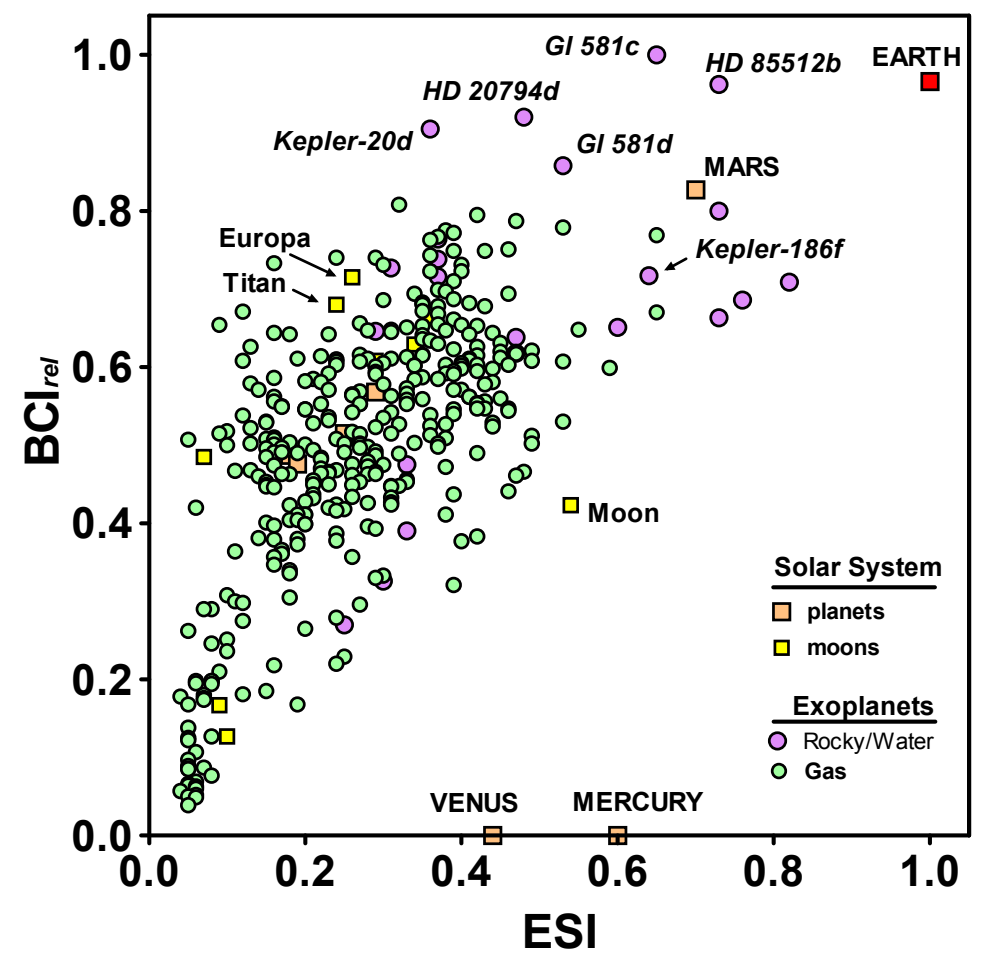

Note: Data current through 14 January 2014.

The BCI should be used with caution, bearing in mind that it does not represent a statistical probability or prediction in any absolute sense of whether complex life has or could evolve on any given planetary body. Rather, it simply provides a tool for the relative assessment of conditions that could be compatible with complex evolutionary scenarios. Solar System bodies are included in the ratings, not only to measure their relative likelihood of being able to host complex organisms 
themselves, but to provide benchmarks for visualizing the nature of exoplanets that are probably more or less likely to harbor complex life than the planets and moons of the Solar System are. As new exoplanets are discovered, new data on known exoplanets is added, or parameters of this template are altered, inclusion of Solar System planets and moons provides a normalizing feature for evaluating the predictive value of the $\mathrm{BCI}_{r e l}$ for any given exoplanet.

A second caution to bear in mind is that the BCI is in one sense overly conservative, and in another sense excessively unconstrained. It is overly conservative in that smaller, Earth-like planets are underrepresented in the sample due to limits on the detection of smaller bodies using current technology. In addition, more data on atmospheric complexity, global layering, and orbital features could raise the BCI of a planetary body as that information becomes available, increasing its apparent likelihood of supporting complex life. On the other hand, the lack of high-resolution detail at such great distances allows for a greater estimate of the possibility for complex life than a more detailed knowledge of the planet might justify. For example, Mars detected at a distance of many light years might appear to have the attributes for supporting complex biological evolution, as reflected in its relatively high BCI; but knowledge of the details of current conditions on Mars [46] and a fuller understanding of its planetary history [13,14,47], which are available to us at close range, provide added constraints which suggest that its BCI overestimates the true prospect of finding complex life on the planet today. As another example, Gliese 581c has the highest BCI of any exoplanet. While its habitability has been questioned because it receives $30 \%$ more radiant energy than Venus [48], and its surface temperature may be much higher than estimated by our model, life on Gliese 581c cannot be ruled out because of uncertainties concerning cloud cover and other details [49] not yet observable at a distance of over 20 light years from Earth.

This paper offers a framework for evaluating the relative potential of other worlds to harbor evolutionary trajectories that have led or could lead to biological complexity. The BCI is a quantitative metric generated from that framework. It is meant to enable a quantitative estimate of the prevalence of complex life in the universe, and to serve as an initial filter for those investigators particularly interested in the emergence of complex life on new exoplanets as they are discovered. An earlier model based on hypothetical assumptions but no empirical data estimated that "only about $0.002 \%$ of all stars in the Milky Way could harbor Earth-analog planets inhabited by complex life" (defined as multicellular organisms capable of photosynthesis) [50]. Our calculation of the BCI based on empirical data for 637 exoplanets shows that $98.4 \%$ of them are rated lower than Europa, and $99.2 \%$ are rated lower than Mars as prospective habitats for the evolution of biological complexity. Europa provides an abode where a moderately complex ecosystem could be thriving today [51-54]. Of the exoplanets considered in this sample, the chance that complex life could have evolved is greater for $1.6 \%$ of them than for Europa.

While our calculations are consistent with the widely-held assumption that the majority of exoplanets have a lower probability of hosting complex life than Europa, even a very low percentage of an extremely large number is high, and it is now recognized that the number of exoplanets is vast. If we assume the existence of 10-100 billion exoplanets in the Milky Way Galaxy, with 1\% of them capable of evolving ecosystems as complex as those that might have existed at some point on Europa, that computes to the possibility of at least 100 million planets that may have seen the evolution of some biological complexity in our galaxy. Thus, this report constitutes the first quantitative argument 
that complex forms of life, while rare in frequency, are likely to be large in absolute number in our galaxy alone, and therefore throughout the Universe.

\section{Methodological Section}

\subsection{Databases}

For data on extrasolar planets, we have relied on the Habitable Exoplanets Catalog (HEC) published online by the Planetary Habitability Laboratory [15] at the University of Puerto Rico at Arecibo. This catalog listed 1,057 confirmed exoplanets as of 14 January 2014, and contained data on all parameters needed to calculate the BCI on 637 of those exoplanets. All subsequent results and discussion are based on this sample of 637 exoplanets, along with the 8 planets and 9 of the largest moons in the Solar System for comparison. It also uniquely lists ESI values for most of the exoplanets. For data on Solar System bodies, the Solar System Dynamics database available online from the Jet Propulsion Laboratory at the California Institute of Technology [55] was used.

\subsection{Calculation of the Biocomplexity Index}

We propose the following calculation for an absolute estimate of the potential for biological complexity $\left(\mathrm{BCI}_{a b s}\right)$ on any planetary body or satellite:

$$
B C I_{a b s}=(S \times E \times T \times G \times A)^{1 / 5}
$$

where $\mathrm{S}$ is a measure of substrate complexity (a function of planetary composition and number of substrate layers plus an index of atmospheric complexity), E is a measure of available energy (summing values for solar flux and redox chemistry), $\mathrm{T}$ is the sum of estimates of the degree to which subsurface and surface temperatures approach an optimum, $\mathrm{G}$ is a measure of geophysical complexity (density plus orbital eccentricity), and A is the relative age of the planetary system.

The values assigned to each of the critical factors are based on objective data available in the HEC database, bearing in mind that much of the information in the database consists of estimates based on models using highly simplified assumptions. Those data include the following:

Substrate (S). The existence of discrete atmospheres, solid substrates, and liquids is known with substantial accuracy for Solar System planetary bodies. Extrapolating this information to extrasolar planets based on their mass/radius ratios provides an acceptable uncertainty of several percent, and serves as the basis for the classification of extrasolar planets as rocky-iron, rocky-water, or gaseous in the HEC database. That information is used to infer the number of distinct layers present on a planetary body, ranging from 1 for bare, rocky planets without an atmosphere, to 3 for planets with solid substrates in contact with an atmosphere and reservoirs of liquid. Rocky planets with an atmosphere but no surface liquids are assigned a value of 2. For those planets with atmospheres, a value of 1 is added for metallic atmospheres (those composed of more than just hydrogen and helium). Bodies deemed to be composed of gasses only are excluded from consideration as habitats for the evolution of complex life. This eliminates $91 \%$ of the 637 exoplanets under consideration as of 14 January 2014. 
Energy (E). The energy factor is obtained by summing values for stellar flux and the potential for redox reactions. Light and chemistry appear to provide the most efficient forms of energy for living systems [2].

Stellar flux is expressed on a scale of 0 to 1 , where 1 indicates an optimal solar flux of two times that on Earth, with a maximum tolerable flux of four times that on Earth. These are arbitrary assumptions, since no empirical evidence is available on what an optimal or maximal tolerable amount of solar energy would be for supporting complex life. The impact of solar flux would depend on many factors besides the intensity of energy flux, such as atmospheric density and composition, surface albedo, surface liquid, cloud cover, etc. It should be noted that a solar flux of slightly more than twice that on Earth has led to a runaway greenhouse heating on Venus, while life on Earth thrives at $50 \%$ of the assumed optimum.

The value for stellar flux is computed as follows: First, the intensity of energy flux is normalized to the flux of solar radiation at $1 \mathrm{AU}$. Then, the final value is calculated as 1 minus the difference between 1 and the ratio of the observed flux to the assumed optimal flux:

$$
\text { Stellar flux }=1-|1-(\mathrm{F} / 2)|
$$

where $\mathrm{F}=$ the observed stellar flux, normalized to the solar flux on Earth. Values above or below twice the solar flux at $1 \mathrm{AU}$ become $<1$, until reaching 0 at a normalized flux of either 0 or 4 .

At an assumed optimal flux of 2 times the solar flux on Earth, Stellar flux $=1-|1-(2 / 2)|=1-|1-1|$ $=1-0=1$, or the optimal possible stellar flux.

A stellar flux either above or below that assumed optimal intensity decreases from 1.0 until reaching 0 at a stellar flux of either 0 or 4 times that of solar flux at 1 AU. Thus, for an assumed stellar flux 4 times the solar flux on Earth, Stellar flux $=1-|1-(4 / 2)|=1-|1-2|=1-1=0$, or an intolerable level of stellar flux.

Likewise, in the total absence of any stellar energy flux, $\quad$ Stellar flux $=1-|1-(0 / 4)|=1-$ $|1-0|=1-1=0$, or a total absence of stellar energy.

Chemical composition is more difficult to ascertain at great distance than stellar flux, but molecules suggesting the possibility of redox reactions have been detected for some exoplanets. Where compounds capable of redox cycling involving $\mathrm{O}_{2}$ - the most potent oxidizer commonly available on Earth - are known to exist, a value of 2 is assigned (or 1.5 if their existence is suspected, for solar system bodies). For a "metals-rich" atmosphere, a value of 1 is assigned, on the assumption that energy-yielding reactions may be possible with such an atmosphere. A hydrogen-rich atmosphere merits a rating of 0.5 . Otherwise, a zero value is assigned.

Temperature (T). Temperatures are not known for any exoplanet. For optically thin atmospheres, observations in the mid-infrared range could in principle reveal the surface temperature, but the required instrumentation is not yet available. The HEC database uses a model for estimating surface temperature, based on the simplified assumptions of an albedo of 0.3 , an atmosphere to planet mass ratio similar to that of Earth, and a greenhouse effect due to $1 \% \mathrm{CO}_{2}$. Equilibrium temperatures are estimated on the assumption of an albedo of 0.3 .

Estimates of thermal optimality are based on the modeled mean planetary equilibrium temperature for the subsurface, and the mean modeled surface temperature for the surface, in the HEC database.

The ratings for thermal optimality are expressed on a scale of 0 to 1 , where 1 indicates an optimal temperature of $312^{\circ} \mathrm{K}$, with a maximum tolerable temperature of $624^{\circ} \mathrm{K}$ assumed. Since no empirical 
evidence is available on what an optimal or maximal tolerable temperature would be for supporting complex life, the optimum is arbitrarily set at the midpoint of the range for liquidity for the most commonly considered solvents for life, with the upper limit set at twice that value. The value of $312{ }^{\circ} \mathrm{K}$ corresponds to the approximate midpoint between the melting point of ammonia ( $195{ }^{\circ} \mathrm{K}$ at 1 bar) and the boiling point of hydrogen peroxide $\left(423{ }^{\circ} \mathrm{K}\right.$ at 1 bar) - two solvents whose ranges of liquidity encompass that for sulfuric acid, hydrocyanic acid, water, and hydrazine - all of which theoretically could be solvents for life [2]. While the selection of $312{ }^{\circ} \mathrm{K}$ is based somewhat arbitrarily on the logic above, it should be noted that this temperature falls very close to the internal temperature maintained by homeothermic animals on Earth.

The factor for thermal optimality is computed from the observed (or modeled) temperature, $\mathrm{T}$, on or beneath the surface, as follows:

$$
\text { Thermal optimality }=1-|1-(\mathrm{T} / 312)|
$$

where $\mathrm{T}=$ the observed or modeled temperature of the planetary body. Values above or below $312{ }^{\circ} \mathrm{K}$ become $<1$, until reaching 0 at either 0 or $624^{\circ} \mathrm{K}$.

For a temperature of $312^{\circ} \mathrm{K}$, Thermal optimality $=1-|1-(312 / 312)|=1-|1-1|=1-0=1$, or the optimal possible temperature.

For a temperature of $624^{\circ} \mathrm{K}$, at which biomolecular stability is unlikely in any solvent at any pressure, Thermal optimality $=1-|1-(624 / 312)|=1-|1-2|=1-1=0$, or a temperature at which complex life is deemed virtually impossible. Likewise, a temperature of absolute zero, where no biological activity is assumed to be possible, leads to a similar conclusion:

Thermal optimality $=1-|1-(0 / 312)|=1-|1-0|=1-1=0$.

Geophysics (G). The square root of planetary density, normalized to that of Earth, constitutes the first value among the geophysical parameters. The square root is used to dampen the effect of this factor. The numerical value for orbital eccentricity is the second geophysical parameter.

Age (A). The age factor is simply the estimated age of an exoplanet's central star (in $10^{9}$ years) divided by 4.5 , to normalize it to the estimated age of the Solar System.

We then compute the relative Biological Complexity Index $\left(\mathrm{BCI}_{r e l}\right)$, which is the ratio of the $\mathrm{BCI}_{a b s}$ value for a planetary body or satellite to the maximum $\mathrm{BCI}_{a b s}$ found in the database. This generates a value ranging from 0 to 1 .

A spreadsheet containing the entire data set and calculations used in computing the BCI are submitted as Supplementary Material.

\section{Conclusions}

A common assumption within the astrobiological community is that life may be relatively pervasive throughout the universe, but in the vast majority of cases is likely to be sequestered, simple, and lacking in diversity [2,30] - the corollary being that the frequency of complex and highly diversified ecosystems on other worlds is likely to be very low [35]. To test this assumption, we have proposed here a Biological Complexity Index (BCI), designed to provide a quantitative estimate of the probability that complex, macro-organismic forms of life could have evolved on other worlds. Our model calculations indicate that complex life may be rare in frequency, but extremely 
abundant in absolute numbers, suggesting the possibility of as many as 100 million planets in our galaxy on which significant biological complexity could have evolved.

\section{Acknowledgments}

Philip von Paris and David Catling provided helpful suggestions during earlier stages of the development of this manuscript.

\section{Author Contributions}

Louis Irwin conceived the idea for the BCI and wrote the first draft of the paper. The three remaining authors contributed to substantial modifications of the original concept and took part in revising the manuscript through several iterations. Abel Méndez curates the Habitable Exoplanets Catalog - the dataset of exoplanets that provided data for all the calculations. He also developed models on which many of the parameters in the dataset are based, and suggested how the calculations should be made. Alberto Fairén is a specialist in planetary history. He contributed in particular to the background section of the paper and critiqued development of the BCI concept at every stage. Dirk Schulze-Makuch specializes in planetary habitability. He was first author of the original paper on Earth similarity and planetary habitability, from which this manuscript was an outgrowth. He critiqued development of the BCI, including details of the calculations, and suggested specific portions of the text throughout.

\section{Conflict of Interest}

The authors declare no conflict of interest.

\section{References}

1. Jones, B.W. Exoplanets-Search methods, discoveries, and prospects for astrobiology. Int. J. Astrobiol. 2008, 7, 279-292.

2. Schulze-Makuch, D.; Irwin, L.N. Life in the Universe: Expectations and Constraints, 2nd ed.; Springer-Verlag: Berlin, Germany, 2008; p. 241.

3. Irwin, L. N.; Schulze-Makuch, D. Cosmic Biology: How Life Could Evolve on Other Worlds, 1st ed.; Praxis: New York, NY, USA, 2011.

4. Hollis, J.M.; Lovas, F.J.; Remijan, A.J.; Jewell, P.R.; Ilyushin, V.V.; Kleine, I. Detection of acetamide (CH3CONH2): The largest interstellar molecule with a peptide bond. Astrophys. J. Lett. 2006, 643, doi:10.1086/505110.

5. Thi, W.-F.; van Zadelhoff, G.-J.; van Dishoeck, E.F. Organic molecules in protoplanetary disks around TTauri and HerbigAe stars. Astron. Astrophys. 2004, 425, 955-972.

6. Pizzarello, S.; Huang, Y.; Becker, L.; Poreda, R.J.; Nieman, R.A.; Cooper, G.; Williams, M. The organic content of the Tagish Lake meteorite. Science 2001, 293, 2236-2239.

7. Sandford, S.A. Terrestrial analysis of the organic component of comet dust. Annu. Rev. Anal. Chem. 2008, 1, 549-578. 
8. Mehringer, D.M.; Snyder, L.E.; Miao, Y.; Lovas, F.J. Detection and confirmation of interstellar acetic acid. Astrophys. J. Lett. 1997, 480, doi:10.1086/310612.

9. Bains, W. Many chemistries could be used to build living systems. Astrobiology 2004, 4, 137-167.

10. Chyba, C.F. Life on other moons. Nature 1997, 385, doi:10.1038/385201a0.

11. Schulze-Makuch, D.; Guan, H.; Irwin, L.N.; Vega, E. Redefining Life: An Ecological, Thermodynamic, and Bioinformatic Approach. In Fundamentals of Life; Elsevier SAS: Amsterdam, The Netherlands, 2002; pp. 169-179.

12. Zolotov, M.Y.; Shock, E.L. Energy for biologic sulfate reduction in a hydrothermally formed ocean on Europa. J. Geophys. Res.-Planets 2003, 108, doi:10.1029/2002JE001966.

13. Schulze-Makuch, D.; Grinspoon, D.H. Biologically enhanced energy and carbon cycling on Titan? Astrobiology 2005, 5, 560-567.

14. Fairén, A.G. A cold and wet Mars. Icarus 2010, 208, 165-175.

15. Méndez, A. Habitable Exoplanets Catalog. Available online: http://www.phl.upr.edu (accessed on 14 January 2014).

16. Udry, S.; Bonfils, X.; Delfosse, X.; Forveille, T.; Mayor, M.; Perrier, C.; Bouchy, F.; Lovis, C.; Pepe, F.; Queloz, D.; Bertaux, J.L. The HARPS search for southern extra-solar planets. Astron. Astrophys. 2007, 469, 43-47.

17. Mayor, M.; Bonfils, X.; Forveille, T.; Delfosse, X.; Udry, S.; Bertaux, J.-L.; Beust, H.; Forveille, T.; Mayor, M.; Perrier, C.; et al. The HARPS search for southern extra-solar planets. An Earth-mass planet in the GJ581 planetary system. Astron. Astrophys. 2009, 507, 487-494.

18. Bonfils, X.; Delfosse, X.; Udry, S.; Forveille, T.; Mayor, M.; Perrier, C.; Bouchy, F.; Gillon, M.; Lovis, C.; Pepe, F.; et al. The HARPS search for southern extra-solar planets XXXI. The M-dwarf sample. Earth Planet. Astrophys.2 011. Available online: http://arxiv.org/abs/1111.5019 (accessed on 22 May 2014).

19. Delfosse, X.; Bonfils, X.; Forveille, T.; Udry, S.; Mayor, M.; Bouchy, F.; Gillon, M.; Lovis, C.; Neves, V.; Pepe, F.; et al. The HARPS search for southern extra-solar planets XXXV. Super-Earths around the M-dwarf neighbors G1433 and Gl667C. Astron. Astrophys. 2012. Available online: http://arxiv.org/abs/1202.2467 (accessed on 22 May 2014).

20. Anglada-Escudé, G.; Arriagada, P.; Vogt, S.; Rivera, E.J.; Butler, R.P.; Crane, J.D.; Shectman, S.A.; Thompson, I.B.; Minniti, D.; Haghighipour, N.; et al. A planetary system around the nearby M-dwarf GJ 667c with at least one super-earth in its habitable zone. Astrophys. J. Lett. 2012, 751, doi:10.1088/2041-8205/751/1/L16.

21. Borucki, W. J.; Agol, E.; Fressin, F.; Kaltenegger, L.; Rowe, J.; Isaacson, H.; Fischer, D.; Batalha, N.; Lissauer, J.J.; Marcy, G.W.; et al. Kepler-62: A five-planet system with planets of 1.4 and 1.6 Earth radii in the habitable zone. Science 2013, 340, 587-590.

22. Tuomi, M.; Anglada-Escudé, G.; Gerlach, E.; Jones, H.R.A.; Reiners, A.; Rivera, E.J.; Vogt, S.S.; Butler, R.P. Habitable-zone super-Earth candidate in a six-planet system around the K2.5V star HD 40307. Astron. Astrophys. 2013, 549, 1-23.

23. Pepe, F.; Lovis, C.; Ségransan, D.; Benz, W.; Bouchy, F.; Dumusque, X.; Mayor, M.; Queloz, D.; Santos, N.C.; Udry, S. The HARPS search for Earth-like planets in the habitable zone. Astron. Astrophys. 2011, 534. Available online: http://arxiv.org/abs/1108.3447 (accessed on 26 May 2014). 
24. Von Paris, P.; Gebauer, S.; Godolt, M.; Grenfell, J.L.; Hedelt, P.; Kitzmann, D.; Patzer, A.B.C.; Rauer, H.; Stracke, B. The extrasolar planet GL 581d: A potentially habitable planet? Astron. Astrophys. 2010. Available online: http://arxiv.org/abs/1009.5814 (accessed on 22 May 2014).

25. Wordsworth, R.D.; Forget, F.; Selsis, F.; Millour, E.; Charnay, B.; Madeleine, J.-B. Gliese 581d is the first discovered terrestrial-mass exoplanet in the habitable zone. Astrophys. J. Lett. 2011, 733. doi:10.1088/2041-8205/733/2/L48

26. Wordsworth, R.D.; Forget, F.; Selsis, F.; Madeleine, J.-B.; Millour, E.; Eymet, V. Is Gliese 581d habitable? Some constraints from radiative-convective climate modeling. Astron. Astrophys. 2010, 522, doi:10.1051/0004-6361/201015053.

27. Kaltenegger, L.; Udry, S.; Pepe, F. A habitable planet around HD 85512? Astron. Astrophys. 2011. Available online: http://arxiv.org/abs/1108.3561 (accessed on 22 May 2014).

28. Schulze-Makuch, D.; Mendez, A.; Fairen, A.G.; von Paris, P.; Turse, C.; Boyer, G.; Davila, A.F.; Antonio, M.R.; Catling, D.; Irwin, L.N. A two-tiered approach to assessing the habitability of exoplanets. Astrobiology 2011, 11, 1041-1052.

29. Ward, P. Life As We Do Not. Know It: The NASA Search for (and Synthesis of) Alien Life, 1st ed.; Viking: New York, NY, USA.

30. Schulze-Makuch, D.; Irwin, L.N. Exotic forms of life in the universe. Naturwissenschaften 2006, 93, 155-172.

31. Rothschild, L.J.; Mancinelli, R.L. Life in extreme environments. Nature 2001, 409, 1092-1101.

32. Thomas, D.N.; Dieckmann, G.S. Antarctic Sea ice-A habitat for extremophiles. Science 2002, 295, 641-644.

33. Lineweaver, C.H.; Davis, T.M. Does the rapid appearance of life on Earth suggest that life is common in the universe? Astrobiology 2002, 2, 293-304.

34. Spiegel, D.S.; Turner, E.L. Bayesian analysis of the astrobiological implications of life's early emergence on Earth. Proc. Natl. Acad. Sci. USA 2012, 109, 395-400.

35. Ward, P.D.; Brownlee, D. Rare Earth: Why Complex Life Is Uncommon in the Universe; Springer-Verlag: New York, NY, USA, 2000; p. 315.

36. Seager, S.; Kuchner, M.; Hier-Majumder, C.A.; Militzer, B. Mass-radius relationships for solid exoplanets. Astrophys. J. 2007, 669, 1279-1297.

37. Fu, R.; O'Connell, R.; Sasselov, D. The interior dynamics of water planets. Astrophys. J. 2010, 708, 1326-1334.

38. Elkins-Tanton, L. Formation of early water oceans on rocky planets. Astrophys. Space Sci. 2011, 332, 359-364.

39. Korenaga, J. On the likelihood of plate tectonics on super-Earths: Does size matter? Astrophys. J. Lett. 2010, 725, doi:10.1088/2041-8205/725/1/L43.

40. Papuc, A.; Davies, G. The internal activity and thermal evolution of Earth-like planets. Icarus 2008, 195, 447-458.

41. Lenardic, A.; Crowley, J.W. On the notion of well-defined tectonic regimes for terrestrial planets in this solar system and others. Astrophysl. J. 2012, 755, doi:10.1088/0004-637X/755/2/132 
42. Quintana, E. V.; Barclay, T.; Raymond, S. N.; Rowe, J.F.; Bolmont, E.; Caldwell, D.A.; Howell, S.B.; Kane, S.R.; Huber, D.; Crepp, J.R.; et al. An Earth-sized planet in the habitable zone of a cool star. Science 2014, 344, 277-280.

43. Heller, R.; Armstrong, J. Superhabitable worlds. Astrobiology 2014, 14, 50-66.

44. Kasting, J.F.; Whitmire, D.P.; Reynolds, R.T. Habitable zones around main-sequence stars. Icarus 1993, 101, 108-128.

45. Grinspoon, D.H. Lonely Planets: The Natural Philosophy of Alien Life, 1st ed.; HarperCollins: New York, NY, USA, 2003; p. 464.

46. Fairén, A. G.; Schulze-Makuch, D.; Rodríguez, A. P.; Fink, W.; Davila, A.; Uceda, E.R.; Furfaro, R.; Amils, R.; McKay, C.P; et al. Evidence for Amazonian acidic liquid water on Mars-A reinterpretation of MER mission results. Planet. Space Sci. 2009, 57, 276-287.

47. Fairén, A. G.; Dohm, J. M.; Baker, V. R.; de Pablo, M.A.; Ruiz, J.; Ferris, J.C.; Anderson, R.C. Episodic flood inundations of the northern plains of Mars. Icarus 2003, 165, 53-67.

48. Von Bloh, W.; Bounama, C.; Cuntz, M.; Franck, S. Habitability of super-Earths: Gliese 581c \& 581d. Proc. Int. Astron. Union 2007, 3, 503-506.

49. Selsis, F.; Kasting, J.; Levrard, B.; Paillet, J.; Ribas, I.; Delfosse, X. Habitable planets around the star G1 581? Astron. Astrophys. 2007, 476, 1373-1387.

50. Bounama, C.; von Bloh, W.; Franck, S. How rare is complex life in the Milky Way? Astrobiology 2007, 7, 745-755.

51. Reynolds, R.T.; Squyres, S.W.; Colburn, D.S.; McKay, C.P. On the habitability of Europa. Icarus 1983, 56, 246-254.

52. Chela-Flores, J. A search for extraterrestrial eukaryotes: Physical and paleontological aspects. Orig. Life Evol. Biosph. 1998, 28, 583-596.

53. Chyba, C.F.; Phillips, C.B. Possible ecosystems and the search for life on Europa. Proc. Natl. Acad. Sci. USA 2001, 98, 801-804.

54. Schulze-Makuch, D.; Irwin, L.N. Alternative energy sources could support life on Europa. EOS Trans. AGU 2001, 82, doi:10.1029/EO082i013p00150.

55. JPL. Solar System Dynamics. Available online: http://ssd.jpl.nasa.gov/ (accessed on 14 January 2014).

(C) 2014 by the authors; licensee MDPI, Basel, Switzerland. This article is an open access article distributed under the terms and conditions of the Creative Commons Attribution license (http://creativecommons.org/licenses/by/3.0/). 